\title{
Optimized Neural Networks-PID Controller with Wind Rejection Strategy for a Quad-Rotor
}

\author{
Chiraz Ben Jabeur ${ }^{1}$, Hassene Seddik ${ }^{2}$ \\ 1,2 Department of Electrical Engineering, University of Tunis, CEREP, ENSIT, Tunis, Tunisia \\ Email: ${ }^{1}$ chirazbenjabeur@gmail.com, ${ }^{2}$ seddik.hassne@gmail.com
}

\begin{abstract}
This study presents a comprehensive modeling and intelligent control strategy for quad-rotor aircraft, a fourrotor unmanned aerial vehicle (UAV). In fact, a PID on-line optimized Neural Networks Approach (PID-NN) has been created for controlling quad-rotor angular trajectories. PID classical controllers, on the other hand, are used to control position, altitude, and speed. The goal of this project is to design a smart Self-Tuning PID controller for attitude angle control that is based on neural networks and capable of supervising a quad-rotor for optimal behavior while tracking a desired trajectory. If the quad-rotor is navigating in hostile environments with irregular disturbances in the form of wind modeled and applied to the overall system, many challenges may arise. The quad-rotor must perform tasks quickly while maintaining stability and accuracy, and it must make decisions quickly in the face of disturbances. This method has a few advantages over traditional control methods like PID controllers. The results of the simulation are based on a comparison of PID and PID-NN controllers based on wind disturbances. These are used to test the quad-behavior rotor's and stability at various levels of strength. These simulation results are satisfactory and show that the proposed PD-NN approach is effective. In fact, the proposed controller has lower errors and a better ability to reject disturbances than the PD controller. It has also proven to be extremely resilient and effective in the face of turbulence in the form of wind disturbances.
\end{abstract}

Keywords-PD Control; PID control; neural networks; hostile environment; quad-rotor; dynamic model; trajectory tracking control; robustness against disturbance

\section{INTRODUCTION}

PID controller has become an essential technical tool and is successfully applied in robotic systems and especially in quad-rotors control strategies [1-10]. Nevertheless, to get ideal control effect, it is required to optimize its three parameters: proportional coefficient $K_{p}$, integral coefficient $K_{i}$, and derivative coefficient $K_{d}$ before placing it into simulation. It is relevant to affirm that many research papers deal with PD and PID parameters optimization with fuzzy logic such as in $[11,12,13]$, but not a lot used neural networks approach.

The aim of parameters optimization is to achieve the best control effect by making the controller and the controlled systems characteristics well-coordinated. If the selected parameters of the PID controller are inopportune, its control effect will be very modest. However, there are all kinds of uncertainties and nonlinearities in the quad-rotor control system, so it is hard to establish the precise traditional math model. Moreover, the traditional parameter optimization methods of the PID controller can't promise the normal work and it is difficult to realize perfect control effect. For this reason, recently, neural networks have demonstrated their growth in PID parameters optimization. In fact, the neural network can learn by itself and simulate the system parameters without knowing about the structure of the system so as to get system rule. Currently, PID controller parameters optimized by neural network are becoming a promising topic $[14,15,16]$, in which, a robust PID controller is presented for flight control of quad-rotor. The proposed PID tuning algorithm continuously adjusts PID parameters which minimize tracking error using artificial neural network.

In the other hand, the controller has an important effect and role in achieving high stability of the quad-rotor In fact, the work focuses on the attitude regulation control problem by maintaining its desired position mainly in presence of external wind disturbances $[17,18]$.

This paper uses artificial intelligence to implement intelligent tracking control on a quad-rotor that is subjected to wind disturbances. In this way, neural networks are used to adjust the parameters of the PD controller for the attitude control in an on-line manner. This attitude control concerns the roll pitch and yaw angles. Thus, there are three neural networks for each angle, which structures are so special and contain at least one hidden layer of two neurons that are the Proportional and the Derivative functions.

Principally, in its fly, the system may encounter some wind disturbances that depend on its environment, and that can affect its trajectory following. In this case, the quad-rotor must compensate these disturbances and continue its navigation without changing its trajectory.

As it is known, flight stability and performance are susceptible to degradation due to wind gusts [19, 20, 21]. Estimating such wind disturbances with sensors and using them to inform flight controls can recover safety. As such, many sensing techniques are dedicated to the measurement of wind in order decrease their effects by control strategies.

Thus, the main objective is the design and implementation of PID classical controllers and PID-NN controllers for controlling the attitude angles, based on neural networks in order to teach the robot a desired behavior while tracking a desired trajectory and facing wind disturbances. The quadrotor must be robust to sudden environment change and must react quickly thanks to the controller and the sensors that give feedback concerning the estimation of wind speed and direction. 
There are eight sections to this paper. The introduction is covered in the first section. The second one is about modeling dynamics. The third section takes into account the wind gust model. The PID classical control strategy and the PID-NN control strategy optimized by neural networks are discussed in the fourth section. Both simulations and results are presented in the fifth section. The analysis is the subject of the sixth section. The conclusion is covered in the eighth section.

\section{DYNAMIC MODEL OF THE QUAD-ROTOR}

Modeling of a quad-rotor is a task of presenting the overall model based on the dynamic models in addition to the DC motor dynamics that must be taken into account. A basic model of an unmanned quad-rotor $[19,20,21]$ is shown in Fig. 1.

Considering that the front and rear motors rotate in the opposite direction of the others. The throttle input is the sum of each motor's thrusts. The pitch movement is achieved by increasing (decreasing) the speed of the rear motor and decreasing (increasing) the speed of the front motor. The roll movement is accomplished in a similar manner by using the lateral motors. The yaw movement is purchased by increasing (decreasing) the speed of the front and rear motors while decreasing (increasing) the speed of the lateral motors.

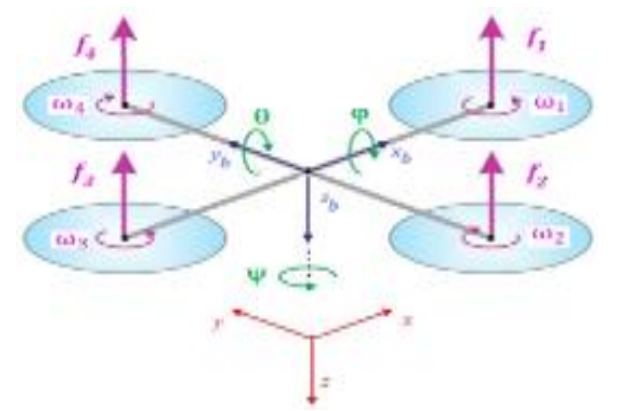

Fig. 1. Model of quad-rotor

\section{A. Newton-Euler Model}

In this section, the specific model information of the quad-rotor architecture based on rigid-body is presented with the application of the Newton-Euler formalism [22].

Two frames have to be considered such as in Fig. 1, the earth inertial frame (E-frame) and the body-fixed frame of the vehicle (B-frame). These are related through three successive rotations; Roll is rotation around the $\mathrm{X}$-axis; Pitch is rotation around the $y$-axis; Yaw is rotation around the $\mathrm{z}$-axis.

The following assumptions have been made in this approach. The origin of the body-fixed frame coincides with the center of mass (COM) of the vehicle body. The axes of the B-frame coincide with the body principal axes of inertia.

The equations of motion are more conveniently formulated in the body-fixed frame because of these reasons [22]: the inertia matrix is time-invariant; advantage of body symmetry can be taken to simplify the equations; measurements taken on-board are easily converted to bodyfixed frame; and control forces are almost always given in body-fixed frame.
The generalized coordinates of the quad-rotor are $q=(x, y, z, \varphi, \theta, \psi)$ where $(x, y, z)$ represents the relative position of the quad-rotor center of mass with respect to an inertial frame $\mathrm{E}$ and $(\varphi, \theta, \psi)$ are the three Euler angles representing the quad-rotor orientation namely the roll, the pitch and the yaw of the vehicle.

Suppose that the transitional and rotational coordinates are in the form: $\xi=(x, y, z) \in \mathfrak{R}^{3}$ and $\eta=(\varphi, \theta, \psi) \in \mathfrak{R}^{3}$.

The total transitional kinetic energy of the quad-rotor is:

$$
T_{\text {trans }}=\frac{1}{2} m \dot{\xi}^{T} \dot{\xi}
$$

Where $m$ is the mass of the quad-rotor.

The rotational kinetic energy is described as

$$
T_{r o t}=\frac{1}{2} J \dot{\eta}^{T} \dot{\eta}=\frac{1}{2} \omega^{T} I \omega
$$

Where matrix $J=J(\eta)$ is the auxiliary matrix, $I$ is the body inertia matrix and $\omega$ is the angular speed.

The potential energy in the system is described as

$$
U=m \cdot g \cdot z
$$

Where $z$ is the quad-rotor altitude and $g$ is the acceleration due to gravity.

The Lagrangian is defined from (1), (2) and (3) as

$$
L=T_{\text {trans }}+T_{\text {rot }}-U
$$

Then the full quad-rotor dynamics is obtained as a function of the external generalized forces $F=\left(F_{\xi}, \tau\right)$ from

$$
F=\frac{d}{d t} \frac{\partial L}{\partial q}-\frac{\partial L}{\partial q}=\left(\begin{array}{c}
m \ddot{x} \\
m \ddot{y} \\
m \ddot{z} \\
I_{x} \ddot{\varphi}+I_{x} \dot{\varphi} \\
I_{y} \ddot{\theta}+I_{y} \dot{\theta} \\
I_{z} \ddot{\psi}+I_{z} \dot{\psi}
\end{array}\right)-\left(\begin{array}{c}
0 \\
0 \\
-m g \\
0 \\
0 \\
0
\end{array}\right)
$$

Where $F_{\xi}$ is the translational force applied to the quad-rotor due to the throttle control input, $\tau$ represents the pitch, roll, and yaw moments.

The principal control inputs are defined as

$$
F_{r}=\left(\begin{array}{lll}
0 & 0 & u
\end{array}\right)^{T}
$$

Where the main thrust is

$$
u=f_{1}+f_{2}+f_{3}+f_{4}
$$

Where $f_{i}=b . \omega_{i}^{2}, b$ is a positive constant and $\omega_{i}$ are the angular speed of the motor $\mathrm{i}$.

Then $F_{\xi}$ can be written as 


$$
F_{\xi}=R \cdot F_{r}
$$

Where $\mathrm{R}$ is the transformation matrix representing the orientation of the quad-rotor as

$$
R=\left[\begin{array}{ccc}
c \theta c \psi & s \theta s \psi & -s \theta \\
c \psi s \theta s \varphi-s \psi s \varphi & s \psi s \theta s \varphi+c \psi c \varphi & c \theta s \varphi \\
c \psi s \theta c \varphi+s \psi s \varphi & s \psi s \theta c \varphi-c \psi s \varphi & c \theta c \varphi
\end{array}\right]
$$

With $c=\cos , s=\sin$

The generalized torques for the $\eta$ variables are

$$
\tau=\left(\begin{array}{lll}
\tau_{\varphi} & \tau_{\theta} & \tau_{\psi}
\end{array}\right)^{T}
$$

Where

$$
\begin{gathered}
\tau_{\varphi}=l\left(f_{3}-f_{1}\right)=b l\left(-\omega_{1}^{2}+\omega_{3}^{2}\right) \\
\tau_{\theta}=l\left(f_{2}-f_{4}\right)=b l\left(-\omega_{2}^{2}+\omega_{4}^{2}\right) \\
\tau_{\psi}=\sum_{i=1}^{1} \tau_{M i}=d\left(-\omega_{1}^{2}+\omega_{2}^{2}-\omega_{3}^{2}+\omega_{4}^{2}\right)
\end{gathered}
$$

Thus, the control distribution from the four actuator motors of the quad-rotor is given by

$$
\left[\begin{array}{c}
u \\
\tau_{\varphi} \\
\tau_{\theta} \\
\tau_{\psi}
\end{array}\right]=\left[\begin{array}{cccc}
b & b & b & b \\
0 & -b l & 0 & b l \\
-b l & 0 & b l & 0 \\
-d & d & -d & d
\end{array}\right]\left[\begin{array}{c}
\omega_{1}^{2} \\
\omega_{2}^{2} \\
\omega_{3}^{2} \\
\omega_{4}^{2}
\end{array}\right]
$$

Where $l$ is the distance from the motors to the centre of gravity, $\tau_{M i}$ is the torque produced by motor $M_{i}$ and $d$ and b are constants known as force-to-moment scaling factor.

The final dynamic model of the quad-rotor looks as

$$
\begin{gathered}
m \ddot{\xi}+\left(\begin{array}{c}
0 \\
0 \\
m g
\end{array}\right)=F_{\xi} \\
J(\eta) \ddot{\eta}+\frac{d}{d t}(J(\eta)) \dot{\eta}-\frac{1}{2} \frac{\partial}{\partial \eta}\left(\dot{\eta}^{T} J(\eta) \dot{\eta}\right)=\tau \\
J(\eta) \ddot{\eta}+\frac{d}{d t}(J(\eta)) \dot{\eta}-\bar{C}(\eta, \dot{\eta})=\tau \\
J(\eta) \ddot{\eta}+C(\eta, \dot{\eta})=\tau
\end{gathered}
$$

Where

$$
F_{\xi}=\left(\begin{array}{c}
c \psi s \theta c \varphi+s \psi s \varphi \\
s \psi s \theta c \varphi-c \psi s \varphi \\
c \theta c \varphi
\end{array}\right) u
$$

And auxiliary Matrix $(J(\eta))=J=T_{\eta}^{T} I T_{\eta}$ with

$$
T_{\eta}=\left(\begin{array}{ccc}
-s \theta & 0 & 1 \\
c \theta s \psi & c \psi & 0 \\
c \theta c \psi & -s \psi & 0
\end{array}\right)
$$

Finally, the dynamic model of the quad-rotor in terms of position $(x, y, z)$ and rotation $(\varphi, \theta, \psi)$ is written as

$$
\left(\begin{array}{c}
\ddot{x} \\
\ddot{y} \\
\ddot{z}
\end{array}\right)=\left(\begin{array}{c}
0 \\
0 \\
-g
\end{array}\right)+\frac{1}{m}\left(\begin{array}{c}
c \psi c \theta c \varphi+s \psi s \varphi \\
s \psi s \theta c \varphi-c \psi s \varphi \\
c \theta c \varphi
\end{array}\right) u
$$

$$
\left(\begin{array}{c}
\ddot{\varphi} \\
\ddot{\theta} \\
\ddot{\psi}
\end{array}\right)=f(\varphi, \theta, \psi)+g(\varphi, \theta, \psi) \tau
$$

Thus, the system has the form of an under-actuated system with six outputs $(x, y, z, \varphi, \theta, \psi)$ and four inputs $\left(u, \tau_{\varphi}, \tau_{\theta}, \tau_{\psi}\right)$

$$
\begin{gathered}
\ddot{x}=(c \psi s \theta c \varphi+s \psi s \varphi) \frac{u}{m} \\
\ddot{y}=(s \psi s \theta c \varphi-C \psi s \varphi) \frac{u}{m} \\
\ddot{z}=-g+(c \theta c \varphi) \frac{u}{m} \\
\ddot{\varphi}=\dot{\theta} \dot{\psi}\left(\frac{I_{y}-I_{z}}{I_{x}}\right)-\frac{J_{p}}{I_{x}} \dot{\theta} \Omega+\frac{\tau_{\varphi}}{I_{x}} \\
\ddot{\theta}=\dot{\psi} \dot{\varphi}\left(\frac{I_{z}-I_{x}}{I_{y}}\right)+\frac{J_{p}}{I_{y}} \dot{\varphi} \Omega+\frac{\tau_{\theta}}{I_{y}} \\
\ddot{\psi}=\dot{\theta} \dot{\varphi}\left(\frac{I_{x}-I_{y}}{I_{z}}\right)+\frac{\tau_{\psi}}{I_{z}}
\end{gathered}
$$

where $I_{x}, I_{y}, I_{z}$ are body inertia, $J_{p}$ is propeller / rotor inertia, and

$$
\Omega=\left(\omega_{1}, \omega_{2}, \omega_{3}, \omega_{4}\right)
$$

\section{B. Actuators Dynamic Modelling: DC-motor}

The driving system of quad-rotors is typically based on armature-controlled DC motors, which are referred to as servo actuators. These actuators provide torque control inputs to train and drive the system. Fig. 2 depicts the DC motor's architecture.

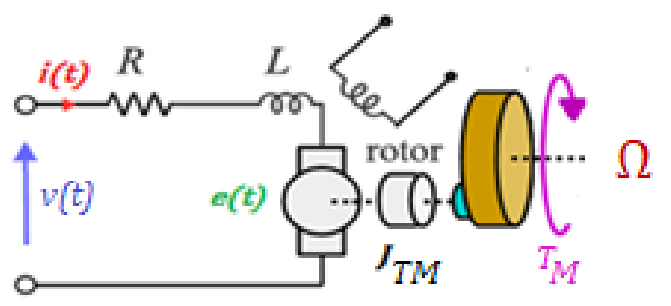

Fig. 2. DC motor Architecture

By applying Kirchhoff's voltage law, it follows that 


$$
\begin{aligned}
v(t) & =V_{R}(t)+V_{L}(t)+e(t) \\
& =R i(t)+L \frac{d i(t)}{d t}+K_{E} \omega_{M}(t)
\end{aligned}
$$

Where $V_{R}(t)$ is the voltage across the resistor $R$ and $V_{L}(t)$ is the voltage across the inductor $L, i(t)$ is the motor current, $K_{E}$ is the motor constant and $\omega_{M}$ is the motor angular speed. Because most robotic motors have low inductance due to design optimization, the inductor effect is ignored.

Therefore, the above equation is simplified according to this one

$$
v(t)=\operatorname{Ri}(t)+K_{E} \omega_{M}(t)
$$

Equation (26) describes the dynamics of the motor

$$
J_{T M} \dot{\omega}_{M}=T_{M}-T_{L}
$$

Where $J_{T M}$ is the total motor moment of inertia, $\dot{\omega}_{M}$ is the motor angular acceleration, $T_{M}$ is the motor torque and $T_{L}$ is the load torque.

The motor torque $T_{M}$ is proportional to the electrical current $i$ through, the above equation becomes

$$
J_{T M} \dot{\omega}_{M}=K_{M} i-T_{L}
$$

Consequently, by combining the above equations, we find

$$
J_{T M} \dot{\omega}_{M}=-\frac{K_{E} K_{M}}{R} \omega_{M}-T_{L}+\frac{K_{M}}{R} v
$$

The two constants $K_{E}$ and $K_{M}$ have the same value even if the units of measurement differ as

$$
P_{E}=P_{M}\left\{\begin{array}{l}
P_{E}=e i=K_{E} i \omega_{M} \\
P_{M}=T_{M} \omega_{\mathrm{M}}=K_{M} \mathrm{i} \omega_{M}
\end{array}\right\} \Rightarrow K_{E}=K_{M}
$$

The real motor system is composed of three units which are the motor itself, the propeller and the gear box. To take the relations between these elements into consideration, some equations must be added:

$$
\begin{gathered}
P_{M} \eta=P_{P} \\
\omega_{M} T_{M P} \eta=\omega_{P} T_{M P}
\end{gathered}
$$

Where: $T_{M P}$ is the motor torque in the propeller axis, $\eta$ is the conversion efficiency of the gear box which attaches the mechanical power of the motor axis $P_{M}$ to the propeller axis one $P_{P}$ and

$$
\begin{gathered}
J_{M} \dot{\omega}_{M}=T_{M}-T_{P M} \\
J_{P} \dot{\omega}_{P}=T_{M P}-T_{P}
\end{gathered}
$$

Where $\omega_{P}$ is the propeller angular speed and $\dot{\omega}_{P}$ is the propeller angular acceleration, $J_{M}$ is the rotor moment of inertia around the motor axis, $J_{P}$ is the rotor moment of inertia around the propeller axis, $T_{M}$ is the propeller torque in the motor axis and $T_{P}$ is the propeller torque.

To calculate the dynamics of the gear box system, this equation is derived as

$$
\left(J_{M}+\frac{J_{P}}{\eta N^{2}}\right) \dot{\omega}_{M}=T_{M}-\frac{T_{P}}{\eta N}
$$

Where $N$ is the reduction ratio of the gear box and is equal to the motor speed $\omega_{M}$ divided by the propeller speed $\omega_{P}$.

According to equation (28), the equations (34) can be rewritten as

$$
\left(J_{M}+\frac{J_{P}}{\eta N^{2}}\right) \dot{\omega}_{M}=-\frac{K_{E} K_{M}}{R} \omega_{M}-\frac{d}{\eta N^{3}} \omega_{M}^{2}+\frac{K_{M}}{R} v
$$

Where $d$ is the aerodynamic drag factor

The motor system can be modeled with the following differential equation.

$$
\left(J_{p}+\eta N^{2} J_{M}\right) \dot{\omega}_{P}=-\frac{K_{E} K_{M}}{R} \eta N^{2} \omega_{P}-d \omega_{P}^{2}+\frac{K_{(3)}}{R}
$$

Where the $\omega_{P}$ coefficient is the total rotational moment of inertia around the propeller axis $J_{T P}$ with

$$
J_{T P}=J_{P}+\eta N^{2} J_{M}
$$

Since the motor differential equation is non-linear, the first order Taylor series method has been adopted to linearize it around its working point as

$$
\dot{\omega}_{P}=A_{P} \omega_{P}+B_{P} v+C_{P}
$$

where $A_{P}$ is the linearized propeller's speed coefficient, $B_{P}$ is the linearized input voltage coefficient and $C_{P}$ is the linearized constant coefficient.

The matrix form of the differential equation is given by

$$
\dot{\Omega}_{P}=A_{P} \Omega_{P}+B_{P} v+C_{P}
$$

Where $\dot{\Omega}_{P}$ is the propellers acceleration vector, $\Omega_{P}$ is the propellers speed vector, and $v$ is the inputs voltage vector.

\section{WIND GUST MODELING}

If a quad-rotor undergoes a crosswind, it may be pushed far downwind or knocked over. Mainly, this leads to forces $f_{v_{i}}$, acting on each rotor. These forces are due to the air flow generated by the wind gust $[23,24,25,26]$. This means that the magnitude of these forces is a function of the additional lateral air flow coming from the wind gust and acting on the propeller as shown in Fig. 3. 


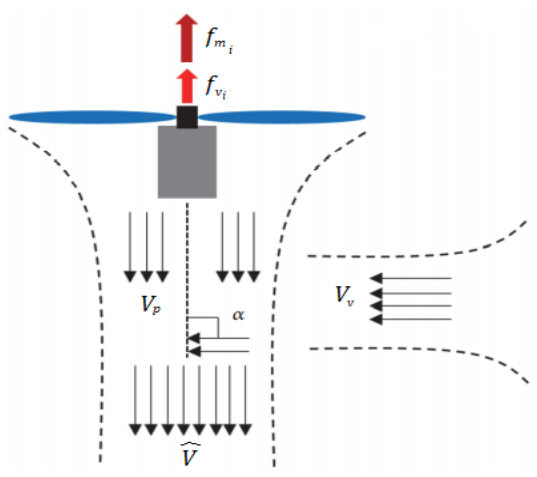

Fig. 3. Aerodynamic analysis of rotor subjected to wind disturbance

Where $f_{m_{i}}=b \omega_{i}^{2}$ represents the thrust of the propellers $\mathrm{i}=1$ 4. $f_{v_{i}}$ designates the additional thrust of the side wind. $\alpha$ symbolizes the angle between the axis of the propeller and the gust of side wind. $V_{v}$ is the speed of the side wind gust. $V_{h}$ is the speed of the wind produced by the propeller. $\widehat{V}$ is the total wind speed.

Therefore, downwind, the total thrust is given by the following expression as

$$
f_{T_{i}}=f_{m_{i}}+f_{v_{i}}=2 \cdot \rho \cdot A \cdot \widehat{V} V_{h}
$$

Where $\rho$ is the air density A is the area of the propeller.

The relation describing the total wind speed is given by

$$
\widehat{V}=\left[\left(V_{v} \cos \alpha+V_{h}\right)^{2}+\left(V_{v} \sin \alpha\right)^{2}\right]^{1 / 2}
$$

When the wind comes from the right of the $\mathrm{x}$-axis, then the angle $\alpha=90^{\circ}$, and this relation becomes

$$
\widehat{V}=\left[V_{h}^{2}+V_{v}^{2}\right]^{1 / 2}
$$

The wind speed $V_{h}$ induced by the propellers is defined as in $[24,26]$ by

$$
\left\|V_{h_{i}}\right\|=\sqrt{\frac{f_{m_{i}}}{2 \cdot \rho \cdot A}}=\sqrt{\frac{d \cdot \omega_{i}^{2}}{2 \cdot \rho \cdot A}}
$$

The thrust produced by the wind will therefore be expressed by

$$
f_{v_{i}}=2 \cdot \rho \cdot A \cdot\left(\frac{d \cdot \omega_{i}^{2}}{2 \cdot \rho \cdot A}+V_{v}^{2}\right)^{1 / 2}-f_{m_{i}}
$$

In addition, the engine torque is opposed by an aerodynamic drag $\tau_{\text {drag }}$. For definition, aerodynamic drag is given by

$$
\tau_{\text {drag }}=\frac{\rho \cdot A}{2} V_{v}^{2}
$$

When the quad-rotor is disturbed by wind gusts, the induced forces $f_{v_{i}}$ in each rotor produce additional aerodynamic wind torques acting on the propellers. These torques could be expressed by

$$
\left[\begin{array}{l}
\tau_{v_{\varphi}} \\
\tau_{v_{\theta}} \\
\tau_{v_{\psi}}
\end{array}\right]=\left[\begin{array}{l}
\left(f_{v_{4}}-f_{v_{2}}\right) l \\
\left(f_{v_{3}}-f_{v_{1}}\right) l \\
4 \\
\sum_{i=1}^{4} \tau_{\text {traîné }}
\end{array}\right]
$$

Where $l$ is the distance between the center of the quad-rotor and the center of the propellers.

On the other hand, the gravitational force applied to the machine is given by

$$
f_{g}=-m \cdot g \cdot \hat{k}
$$

Where $\hat{k}$ denotes the unit vector coinciding with the $\mathrm{z}$ axis and $g$ refers to the acceleration of gravity.

Consequently, we have

$$
\left\{\begin{array}{l}
F=R\left(\sum_{i=1}^{4} f_{M_{i}}+\sum_{i=1}^{4} f_{v_{i}}\right)+f_{g} \\
\tau=\tau_{A}+\tau_{v A}
\end{array}\right.
$$

Where $\tau_{A}=\left[\tau_{\varphi}, \tau_{\theta}, \tau_{\psi}\right]^{T}$ and $\tau_{v A}=\left[\tau_{v_{\varphi}}, \tau_{v_{\theta}}, \tau_{v_{\psi}}\right]^{T}$.

From (14) and (17), we have

$$
\left\{\begin{array}{c}
m \ddot{\xi}=R\left(\sum_{i=1}^{4} f_{M_{i}}+\sum_{i=1}^{4} f_{v_{i}}\right)+f_{g} \\
J \dot{\Omega}=-\Omega \wedge J \Omega+\tau_{A}+\tau_{v A}-\tau_{f a}-\tau_{g h}
\end{array}\right.
$$

This leads to

$$
\left\{\begin{array}{c}
\ddot{x}=-\frac{K_{t x}}{m} \dot{x}+(c \psi s \theta \cos \varphi+\sin \psi \sin \varphi) \frac{U_{1}}{m}+W_{1} \\
\ddot{y}=-\frac{K_{t y}}{m} \dot{y}+(\sin \psi \sin \theta \cos \varphi-\cos \psi \sin \varphi) \frac{U_{1}}{m}+W_{1} \\
\ddot{z}=-\frac{K_{t y}}{m} \dot{z}-g+(\cos \theta \cos \varphi) \frac{U_{1}}{m}+W_{1} \\
\ddot{\varphi}=-\dot{\theta} \dot{\psi}\left(\frac{I_{z}-I_{y}}{I_{x}}\right)-\frac{J_{r}}{I_{x}} \Omega_{r} \dot{\theta}-\frac{K_{f a x}}{I_{x}} \dot{\varphi}^{2}+\frac{U_{2}}{I_{x}}+W_{2} \\
\ddot{\theta}=\dot{\varphi} \dot{\psi}\left(\frac{I_{z}-I_{x}}{I_{y}}\right)+\frac{J_{r}}{I_{y}} \Omega_{r} \dot{\varphi}-\frac{K_{f a y}}{I_{y}} \dot{\theta}^{2}+\frac{U_{3}}{I_{y}}+W_{3} \\
\ddot{\psi}=-\dot{\theta} \dot{\varphi}\left(\frac{I_{y}-I_{x}}{I_{z}}\right)-\frac{K_{f a z}}{I_{z}} \dot{\psi}^{2}+\frac{U_{4}}{I_{z}}+W_{4}
\end{array}\right.
$$

Where

$$
U=\left[U_{1}, U_{2}, U_{3}, U_{4}\right]=\left[\begin{array}{c}
b\left(\omega_{1}^{2}+\omega_{2}^{2}+\omega_{3}^{2}+\omega_{4}^{2}\right) \\
b l\left(\omega_{4}^{2}-\omega_{2}^{2}\right) \\
b l\left(\omega_{3}^{2}-\omega_{1}^{2}\right) \\
d\left(\omega_{1}^{2}-\omega_{2}^{2}+\omega_{3}^{2}-\omega_{4}^{2}\right)
\end{array}\right]=\left[\begin{array}{c}
u \\
\tau_{\varphi} \\
\tau_{\theta} \\
\tau_{\psi}
\end{array}\right]
$$

Where $W=\left[W_{1}, W_{2}, W_{3}, W_{4}\right]$ is the vector disturbances produced by the wind and is defined as 


$$
W=\left[\begin{array}{c}
\sum_{i=1}^{4} \frac{f_{v_{i}}}{m} \\
\frac{\left(f_{v_{4}}-f_{v_{2}}\right) l}{I_{x}} \\
\frac{\left(f_{v_{3}}-f_{v_{1}}\right) l}{I_{y}} \\
\frac{\sum_{i=1}^{4} \tau_{\text {traîné }}}{I_{z}}
\end{array}\right]
$$

These disturbances are applied to the quad-rotor in order to test the system control performances. The major problem is that the quad-rotor must overcome these disturbances and must not be blown by the wind whatever its strength. Evidently, the relation between the weight of the quad-rotor and the speed wind must be taken into account and respected.

\section{CONTROL STRATEGIES}

In this section, a trajectory tracking hindrance and a position control problem are presented when the quad-rotor is evolving in a hostile environment.

The aim of the work is based on two control strategies: a PID classical control strategy and a PID-NN intelligent control strategy, a comparative study will be done based on the robustness of these controllers to the disturbances caused by the wind.

\section{A. PID Control strategies}

The PID control strategy is presented in Fig.4. Two types of PD controllers are adopted: the first one concern positions and altitude control, the second one is opted for attitude angles control, and the PID controller is devoted for the motors control.

The continuous transfer function of a traditional PID controller is represented as

$$
C(p)=K_{P}+\frac{K_{I}}{p}+K_{D} p
$$

Where $K_{P}$ is the proportional coefficient, $K_{I}$ is integral coefficient and $K_{D}$ is the derivative coefficient.

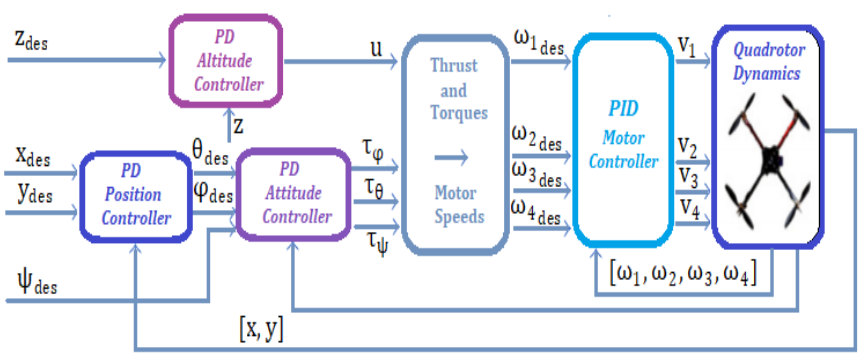

Fig. 4. PD and PID control strategies

The control law expression is based on the error between the desired variable and the process variable and is given by

$$
u(t)=K_{P} e(t)+K_{I} \int e(t) d t+K_{D} \frac{d e(t)}{d t}
$$

The PD controllers for positions: $\mathrm{x}, \mathrm{y}$ and $\mathrm{z}$ respectively are described as

$$
\begin{aligned}
& u_{x}=K_{P x}\left(x_{d e s}-x\right)+K_{D^{x}}\left(\dot{x}_{\text {des }}-\dot{x}\right) \\
& u_{y}=K_{P y}\left(y_{d e s}-y\right)+K_{D y}\left(\dot{y}_{d e s}-\dot{y}\right) \\
& u_{z}=K_{P z}\left(z_{d e s}-z\right)+K_{D z}\left(\dot{z}_{d e s}-\dot{z}\right)
\end{aligned}
$$

The PD controllers for angles (Altitude and Attitude), Roll ' $\varphi$ ', pitch ' $\theta$ ' and yaw ' $\psi$ ', respectively are described as

$$
\begin{gathered}
u_{\varphi}=K_{P \varphi}\left(\varphi_{d e s}-\varphi\right)+K_{D \varphi}\left(\dot{\varphi}_{\text {des }}-\dot{\varphi}\right) \\
u_{\theta}=K_{P \theta}\left(\theta_{d e s}-\theta\right)+K_{D \theta}\left(\dot{\theta}_{d e s}-\dot{\theta}\right) \\
u_{\psi}=K_{P \psi}\left(\psi_{d e s}-\psi\right)+K_{D \psi}\left(\dot{\psi}_{d e s}-\dot{\psi}\right)
\end{gathered}
$$

The PID controllers for motors speeds are respectively described as

$$
u_{\omega 1}=K_{p \omega 1}\left(\omega_{1 d e s}-\omega_{1}\right)+K_{I \omega 1} \int\left(\omega_{1 d e s}-\omega_{1}\right) d t+K_{D^{\omega 1}}\left(\dot{\omega}_{1 d e s}-\dot{\omega}_{1}\right)
$$

$$
u_{\omega 2}=K_{p \omega 2}\left(\omega_{2 d e s}-\omega_{2}\right)+K_{I \omega 2} \int\left(\omega_{2 d e s}-\omega_{2}\right) d t+K_{D \omega 2}\left(\dot{\omega}_{2 d e s}-\dot{\omega}_{2}\right)
$$

$$
u_{\omega 3}=K_{p \omega 3}\left(\omega_{3 d e s}-\omega_{3}\right)+K_{I \omega 3} \int\left(\omega_{3 d e s}-\omega_{3}\right) d t+K_{D^{\omega 3} 3}\left(\dot{\omega}_{3 d e s}-\dot{\omega}_{3}\right)
$$

$$
u_{\omega 4}=K_{P \omega 4}\left(\omega_{4 d e s}-\omega_{4}\right)+K_{I \omega 4} \int\left(\omega_{4 d e s}-\omega_{4}\right) d t+K_{D \omega 4}\left(\dot{\omega}_{4 d e s}-\dot{\omega}_{4}\right)
$$

The Ziegler and Nichols approach is used to tune the PID coefficients when using a closed loop scheme [27, 28, 29]. The simplicity of this method is its main advantage. It consists in setting the integral $K_{I}$ gain to the maximum, and derivative $K_{D}$ gain to zero.

However, the proportional $K_{P}$ gain is then increased until it reaches the critical gain $K_{\text {osc }}$, and the system oscillates continuously among constant amplitude oscillations with $T_{\text {osc }}$ period. Then $K_{o s c}$ and $T_{o s c}$ are used to set the PID gains based on mathematical approximations [27, 28, 29].

\section{B. PID Optimized Neural Networks Approach: PID-NN Control strategy}

a) Neural network controller scheme

The main idea is the parameters optimization of the PID controller dedicated for the attitude angles, with the use of the famous feed-forward neural network. The methodology principle is to dynamically and on line adjust these 
parameters in order to reach the optimal PID-NN controller performances according to the system running state.

The control strategy is based on three groups of controllers, one PD controller group for the positions and the altitude, one PD-NN controller group for the attitude angles and one PID controller group for the speed of the quad-rotor.

The principle of the PD optimized neural networks approach for the attitude angles is shown in Fig. 5.

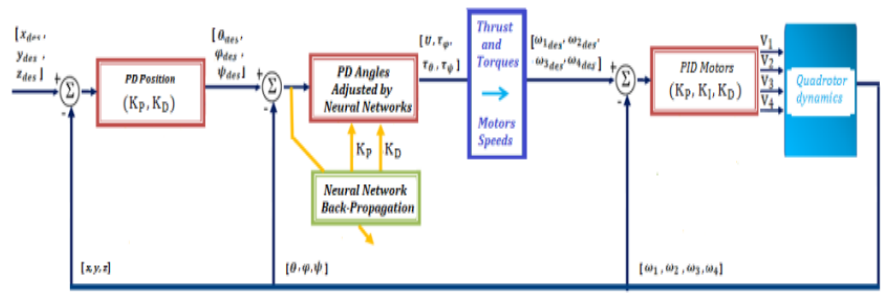

Fig. 5. PD and PID control strategies with Neural Network Back Propagation

\section{b) Neural network architecture}

The neural networks used are multi-layer networks with the back-propagation training method. In this work, three neural networks were designed for attitude angles: Roll angle, Pich angle and Yaw angle.

For each angle, as is seen in the Fig. 5, the structure of the neural network is composed of three layers. The input layer contains the actual and the desired angles, the hidden layer is composed of two hidden neurons that are the proportional and the derivative functions and the third layer deals with the torques corresponding to each angle [21].

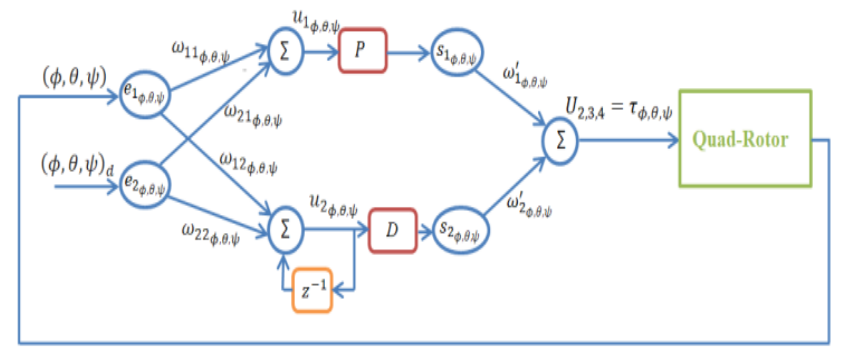

Fig. 6. PD and PID control strategies

The mathematical equations that govern the principle of these three similar neural networks are described in layers as follows.

$\checkmark$ The input layer with two input neurons

$$
\left\{\begin{array}{c}
e_{1(\varphi, \theta, \psi)}(k)=(\varphi, \theta, \psi)(k) \\
e_{2(\varphi, \theta, \psi)}(k)=(\varphi, \theta, \psi)_{d}(k)
\end{array}\right.
$$

With k represents the iterations.

$\checkmark$ The hidden layer composed of two hidden neurons and whose inputs are described by

$$
u_{j(\varphi, \theta, \psi)}(k)=\sum_{i=1}^{2} \omega_{i j(\varphi, \theta, \psi)}(k) e_{i(\varphi, \theta, \psi)}(k), j=1,2
$$

Where $\omega_{i j}(k)$ are the weights between the hidden and the input layers.
So as:

$$
\left\{\begin{array}{l}
u_{1_{\phi, \theta, \psi}}(k)=\omega_{11_{\phi, \theta, \psi}}(k) \cdot e_{1_{\phi, \theta, \psi}}(k)+\omega_{21_{\phi, \theta, \psi}}(k) \cdot e_{2_{\phi, \theta, \psi}}(k) \\
u_{2_{\phi, \theta, \psi}}(k)=\omega_{12_{\phi, \theta, \psi}}(k) \cdot e_{1_{\phi, \theta, \psi}}(k)+\omega_{22_{\phi, \theta, \psi}}(k) \cdot e_{2_{\phi, \theta, \psi}}(k)
\end{array}\right.
$$

$\checkmark$ The outputs of the hidden layer are described with:

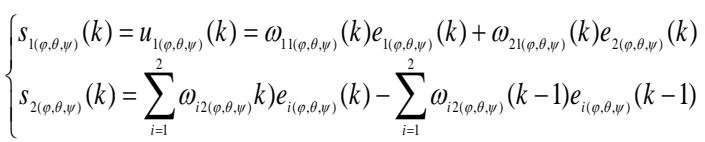

$\checkmark$ The output layer has a single neuron whose main function is to provide the control signal. This neuron is expressed by:

$$
\begin{aligned}
\left(U_{2}, U_{3}, U_{4}\right)(k)= & \omega_{1_{\phi, \theta, \psi}}^{\prime}(k) \cdot s_{1_{\phi, \theta, \psi}}(k) \\
& +\omega_{2_{\phi, \theta, \psi}}^{\prime}(k) \cdot s_{2_{\phi, \theta, \psi}}(k)
\end{aligned}
$$

With $\omega_{j}^{\prime}$ are the weights between the output and the hidden layers.

This gives

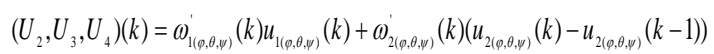

Finally

$$
\begin{aligned}
& \left(U_{2}, U_{3}, U_{4}\right)(k)=K_{P}\left(\omega_{11(\varphi, \theta, \psi)}(k) e_{1(\varphi, \theta, \psi \psi}(k)+\omega_{2\lfloor(\varphi, \theta, \psi)}(k) e_{2(\varphi, \theta, \psi)}(k)\right)
\end{aligned}
$$

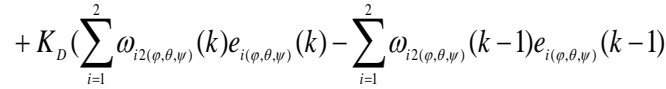

Initially, the weights between the input layer and the hidden layer are chosen as such:

$$
\left\{\begin{array}{l}
\omega_{1 j_{(\varphi, \theta, \psi)}}=+1 \\
\omega_{2 j_{(\varphi, \theta, \psi)}}=-1
\end{array} \quad j=1,2\right.
$$

And the weights between the hidden layer and the output layer are defined, through the principle of the classical PD controller, by

$$
\left\{\begin{array}{l}
\omega_{1_{(\varphi, \theta, \psi)}^{\prime}}^{\prime}=K_{p} \\
\omega_{2_{(\varphi, \theta, \psi)}^{\prime}}^{\prime}=K_{d}
\end{array}\right.
$$

The algorithm used as auto-tuning is the back-propagation training algorithm, chosen for its ability to adapt to changing environments. The back-propagation algorithm trains the neural network and updates the PD constants online. This algorithm is based on the gradient descent method and modifies the weights to minimize the cost function $\mathrm{E}(\mathrm{k})$, which is defined as the square of the difference between the input to the network and the desired input.

$$
E(k)=\frac{1}{2} e_{(\varphi, \theta, \psi)}^{2}(k)
$$

Where $e_{(\varphi, \theta, \psi)}$ are the errors between the attitude angles and their desired values. Variable $e_{(\varphi, \theta, \psi)}(k)$ can be described as

$$
e_{(\varphi, \theta, \psi)}(k+1)=e_{(\varphi, \theta, \psi)}(k)+\Delta e_{(\varphi, \theta, \psi)}(k)
$$


At first, the entries should be applied to the network; it propagates from the first layer to the hidden layers, to output the angles of attitude. These angles are then compared to the desired values and a corresponding error vector is calculated. These errors propagate back, from the output layer, to all the neurons of the hidden layer so that all the neurons in the network have received an error signal thus involving it in its contribution to the total error.

The weights between the hidden layer and the output layer, which are the $K_{P}$ and $K_{D}$ coefficients of the PD controller, are updated by the following equation:

$$
\omega_{j}^{\prime}(k+1)=\omega_{j}^{\prime}(k)-\eta_{j} \cdot \Delta \omega_{j}^{\prime}(k)
$$

With $\eta_{j}$ is the learning rate and $\Delta \omega_{j}^{\prime}(k)$ is the error gradient at unit $j$.

The variable quantity $\Delta e_{(\varphi, \theta, \psi)}(k)$ is defined as:

$$
\Delta e_{(\varphi, \theta, \psi)}(k)=\frac{\partial e_{(\varphi, \theta, \psi)}(k)}{\partial \omega_{j}^{\prime}(k)} \Delta \omega_{j}^{\prime}(k)
$$

With this algorithm, the gradient is updated by the following expression as

$$
\begin{aligned}
& \Delta \omega_{j}^{\prime}(k)=-\eta \frac{\partial E(k)}{\partial \omega_{j}^{\prime}(k)}=-\eta \frac{1}{2} \frac{\partial e_{(\varphi, \theta, \psi)}^{2}(k)}{\partial \partial \omega_{j}^{\prime}(k)} \\
& =-\eta e_{(\varphi, \theta, \psi)}(k) \frac{\partial e_{(\varphi, \theta, \psi)}(k)}{\partial \omega_{j}^{\prime}(k)} \\
& =-\eta \frac{\partial E(k)}{\partial U_{2,3,4}(k)} \cdot \frac{\partial U_{2,3,4}(k)}{\partial \omega_{j}^{\prime}(k)}
\end{aligned}
$$

Giving

$$
\begin{array}{r}
\Delta \omega_{j}^{\prime}(k)=-\eta e_{(\varphi, \theta, \psi)}(k) \cdot s_{j}(k) \cdot \frac{\partial(\varphi, \theta, \psi)(k)}{\partial U_{2,3,4}(k)} \\
=-e_{\varphi, \theta, \psi}(k) \cdot s_{j}(k) \cdot \delta(k)
\end{array}
$$

Where

$$
\delta(k)=\operatorname{sgn} \frac{(\varphi, \theta, \psi)(k)-(\varphi, \theta, \psi)(k-1)}{U_{2,3,4}(k)-U_{2,3,4}(k-1)}
$$

From the input layer to the hidden layer, the weights between the input layer and the hidden layer are updated by the following equation

$$
\omega_{i j}(k+1)=\omega_{i j}(k)-\eta_{j} . \Delta \omega_{i j}(k)
$$

With $\eta_{j}$ is the learning rate and $\Delta \omega_{i j}(k)$ is the error gradient at unit $j$, and

$$
\begin{aligned}
& \Delta \omega_{i j}(k)=\frac{\partial E(k)}{\partial \omega_{i j}(k)} \\
& =\frac{\partial E(k)}{\partial U_{2,3,4}(k)} \cdot \frac{\partial U_{2,3,4}(k)}{\partial s_{j}(k)} \cdot \frac{\partial s_{j}(k)}{\partial u_{j}(k)} \cdot \frac{\partial u_{j}(k)}{\partial \omega_{j}^{\prime}(k)} \\
& \Delta \omega_{i j}(k)=-e_{(\varphi, \theta, \psi)}(k) \cdot \omega_{j}^{\prime}(k) \cdot e_{i}(k) \cdot \delta(k)
\end{aligned}
$$

\section{SIMULATION RESULTS}

\section{A. Controlled quad-rotor parameters responses with PD} and PD-NN controllers

The training design of the PID and PID-NN controllers is done with the Matlab/Simulink environment, and simulation results are carried out with considering wind disturbances applied in three directions. The control strategy consists on a desired trajectory which is founded on $\mathrm{xd}$, yd and $\mathrm{zd}$ desired positions and also on desired yaw angle $\psi_{\mathrm{d}}=\mathrm{pi} / 6$. They are presented in Fig. 7.
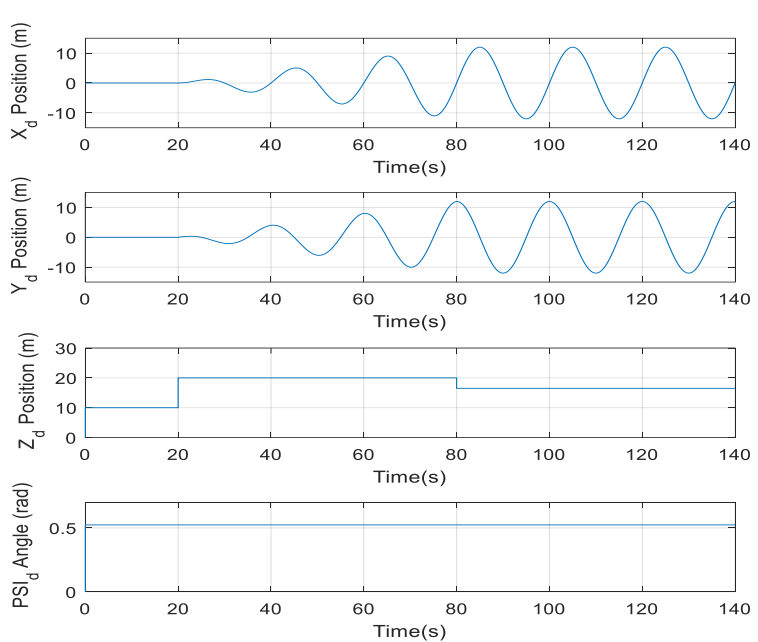

Fig. 7. $\mathrm{x}, \mathrm{y}$ and $\mathrm{z}$ positions and $\psi$ angle references

Fig. 8, Fig. 9, Fig. 10 represent results of all controlled quad-rotor parameters compared to references for both training control strategies PD and PD-NN controllers.
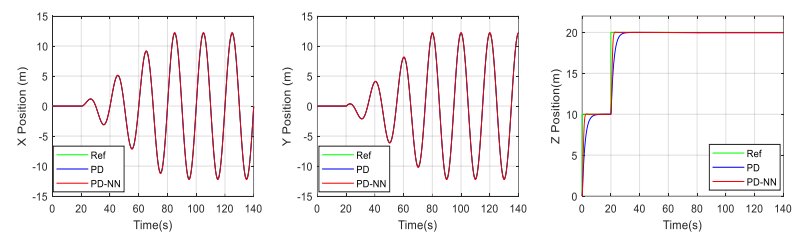

Fig. 8. Controlled $\mathrm{x}, \mathrm{y}$ and $\mathrm{z}$ positions
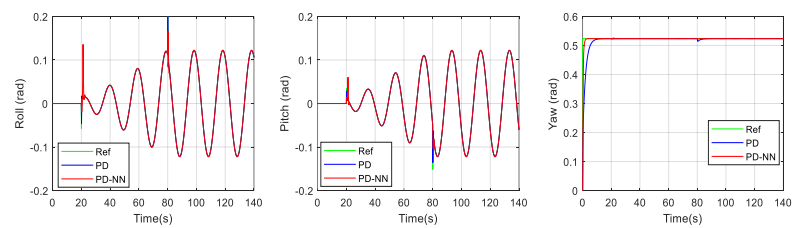

Fig. 9. Controlled Roll, Pitch and Yaw angles
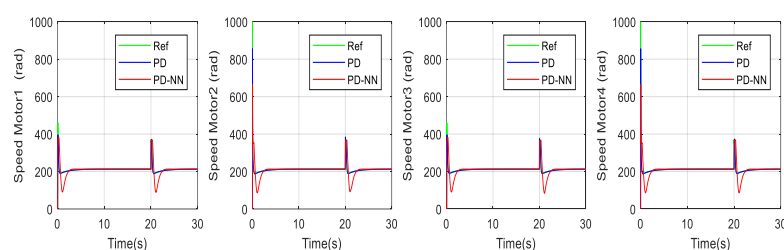

Fig. 10. Controlled Motors Speeds

The trajectory tracking is given in Fig. 11. 


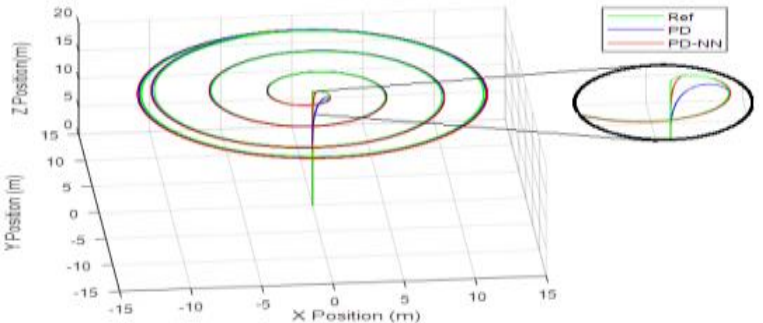

Fig. 11. Spiral Trajectory of Quad-rotor

\section{B. $P D$ and $P D-N N$ controller comparative study with wind disturbances}

The goal is to compare PD and PD-NN control strategies facing different levels of wind disturbances to test the robustness and the stability of both approaches against these troubles. Some wind velocities $V_{v}$ were applied increasingly and two different stability thresholds were obtained for both control strategies. In fact, a comparative study is conducted and simulations results were obtained at what times several wind acceleration values were applied. The stability test is verified on the trajectory tracking and how the control strategy is able to let the quad-rotor following the reference trajectory.

Some simulations results are exposed for selected wind disturbances:

\section{$V_{v}=3.24$ knots}

The trajectory tracking is represented in Fig. 12.

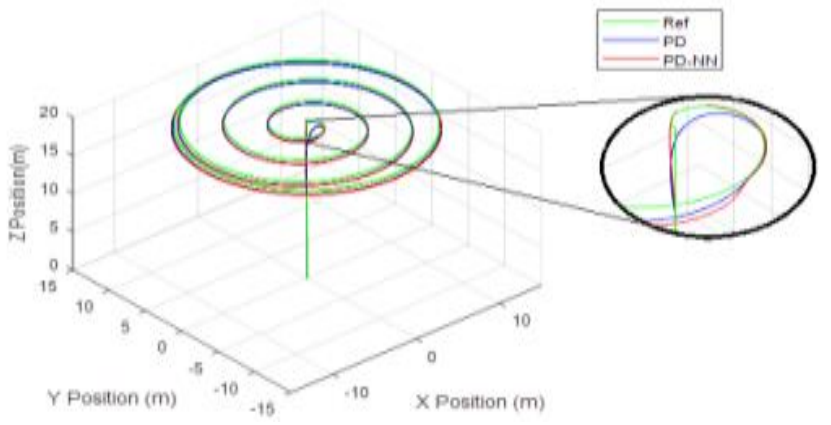

Fig. 12. Spiral Trajectory of Quad-rotor

\section{$V_{v}=9.18$ knots}

The trajectory tracking is represented in Fig. 13.

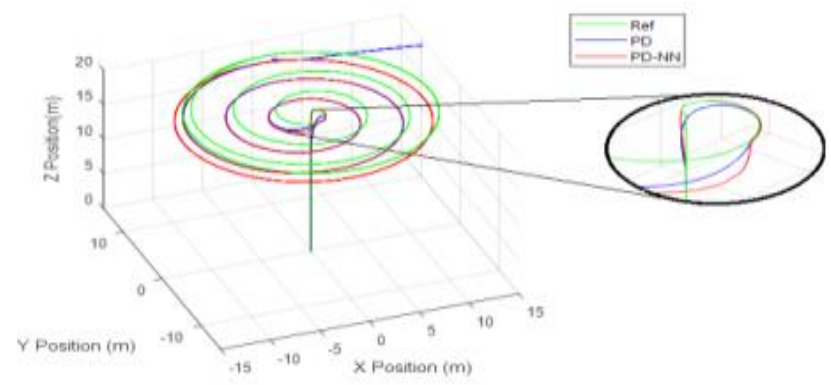

Fig. 13. Spiral Trajectory of Quad-rotor

$V_{v}=10.25$ knots. $s^{-1}$

The trajectory tracking is represented in Fig. 14.

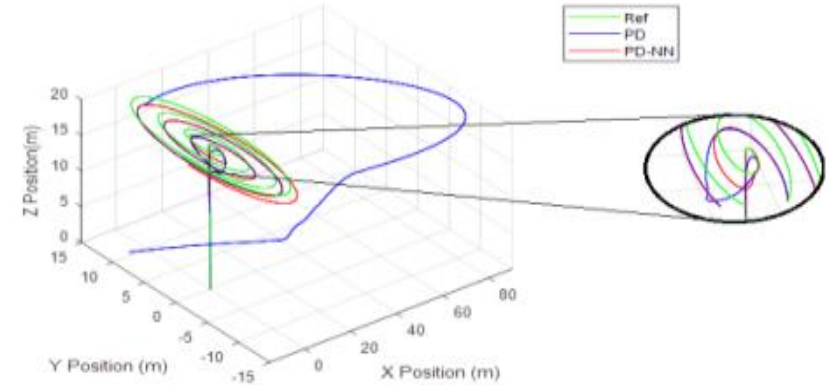

Fig. 14. Spiral Trajectory of Quad-rotor

$$
V_{v}=21.06 \text { knots }
$$

The trajectory tracking is represented by:

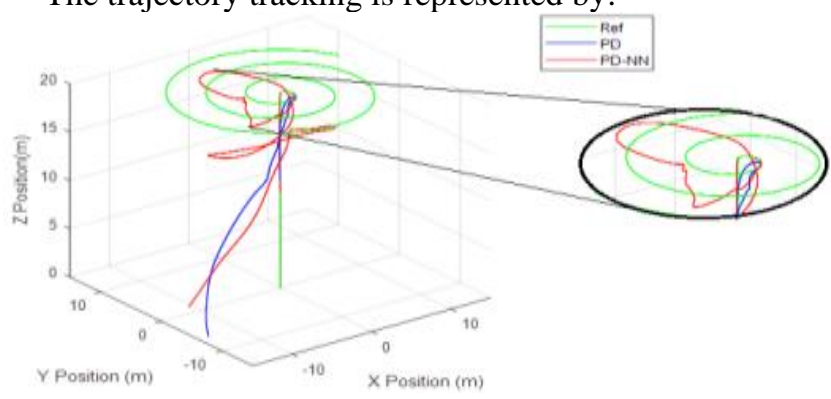

Fig. 15. Spiral Trajectory of Quad-rotor

To represent the variations of the quad-rotor positions with regards to the disturbances caused by the wind velocities, Fig. 16 highlights the stability thresholds of both control strategies.

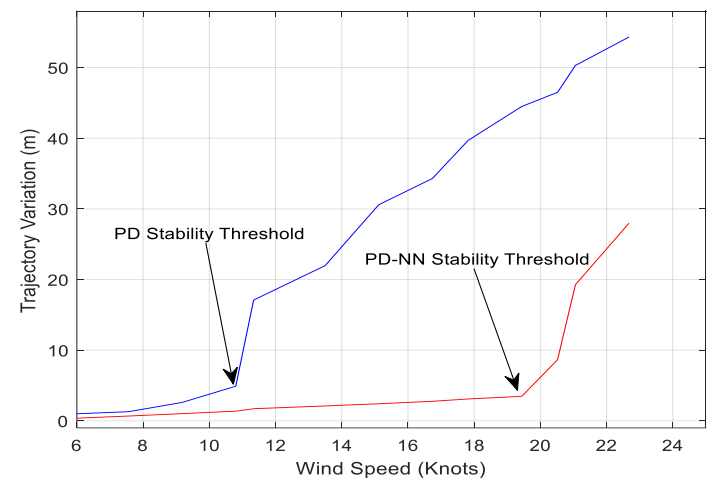

Fig. 16. Quad-rotor trajectory variations against wind disturbances

\section{C. $\quad P D$ and $P D-N N$ strategies for trajectories tracking}

It is a task of varying the trajectories types in order to check the efficiency of both strategies to follow desired ones. A spiral trajectory is kept with rise and fall protocols. Fig. 17 shows, by simulations, the behavior of each control strategy.

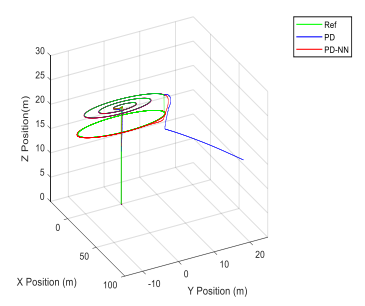

(a)

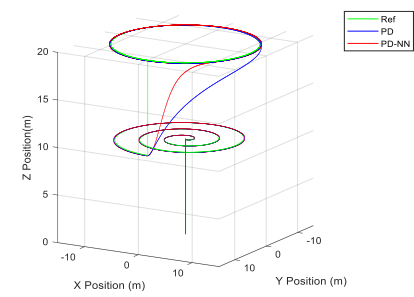

(b)
Fig. 17. (a) Quad-rotor medium fall from $20 \mathrm{~m}$ to $16 \mathrm{~m}$, (b) Quad-rotor very high rise from $10 \mathrm{~m}$ to $20 \mathrm{~m}$ 


\section{DISCUSSION AND ANALYSES}

In the previous section, desired behaviors were applied to the quad-rotor and the controlled parameters responses were exposed taking into account the application and the variation of wind acceleration disturbances. Evidently, in all the previous cited cases, the quad-rotor should behave exactly like the references with both control strategies.

In fact, from Fig. 8 to Fig. 10, the quad-rotor follows the desired positions, angles and motors speeds with both controllers without adding wind disturbance.

In Fig. 11, the quad-rotor follows the desired trajectory. It is to confirm that the trajectory selected is very hard to track especially when the system is highly instable. In fact, this trajectory is composed of straight sections along $\mathrm{z}$ direction and compound alternations along $\mathrm{x}$ and $\mathrm{y}$ which lead to a circular and spiral trajectory.

In addition, it should be mentioned that the PID-NN controller offers faster and quick responses as represented in Fig. 18.

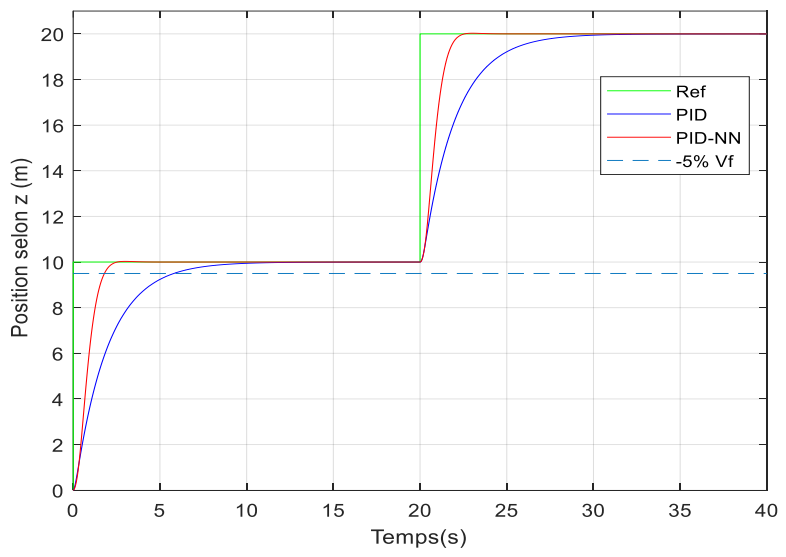

Fig. 18. PID and PID-NN controllers times responses at 5\%

According to this figure, time responses at 5\% are as for PID-NN controller $\tau_{r_{5} \%}=1.8 \mathrm{~s}$, For PID controller $\tau_{r_{5 \%}}=$ $5.8 \mathrm{~s}$. However, quad-rotor tests show fairly acceptable errors. The following table shows the maximum values of the errors reached for positions and angles for both strategies the classical one and the intelligent one.

When applying wind disturbances, the stability and the robustness of both control strategies were tested. Fig. 12 to Fig. 15 shows the behavior of the proposed controllers facing wind disturbances. Effectively, when increasing disturbances, the controlled quad-rotor with PD and PD-NN controllers loses stability, however the second controller proposes better responses and the stability threshold is higher if compared with the PD controller.

TABLE I. ERRORS VALUES FOR POSITIONS AND ANGLES FOR BOTH STRATEGIES

\begin{tabular}{|c|c|c|c|c|c|c|}
\hline Strategy & $\boldsymbol{x}_{\text {pos }}$ & $\boldsymbol{y}_{\text {pos }}$ & $\boldsymbol{z}_{\text {pos }}$ & $\boldsymbol{\varphi}$ & $\boldsymbol{\theta}$ & $\boldsymbol{\psi}$ \\
\hline PID & 0.2596 & 0.2597 & 0.0580 & 0.1287 & 0.0279 & 0.0096 \\
\hline PID-NN & 0.2048 & 0.2047 & 0.0451 & 0.0569 & 0.0145 & 0.0014 \\
\hline
\end{tabular}

Fig. 16 shows the trajectory variation against disturbance cause by the wind acceleration. It is to notice that the controlled PD quad-rotor reaches instability from wind acceleration of 10.92 knots, but with the PD-NN, it reaches instability from wind acceleration of 19.65 knots. Fig. 18 proves that the PD-NN controllers allow better responses than the PD one in terms of stability and rapidity.

Concerning trajectory tracking, it is observed that the controlled PID quad-rotor can't follow all types of trajectories in cases of falling down. Nevertheless, the controlled PD-NN quad-rotor can track all types of trajectories.

Basically, the PID-NN controller allows improved responses whether in the case of applying wind disturbances with different degrees: it seems to have a large marge of stability against these disturbances. Or in the case of trajectory tracking: it follows all the trajectories without any constraint. It might also be noted that in hover state and in presence of wind disturbances, the quad-rotor can maintain its attitude. This case demonstrates its stability and robustness.

\section{CONCLUSION}

In this paper, a PID and a PID-NN control schemes were proposed for a quad-rotor that is evolving in an environment with external influences. The work sorts out the problem of wind disturbances and proposes a solution to this problem based on the PID-NN controller.

Moreover, the used trajectory is very difficult to follow, but the PID-NN controller optimized by neural networks leads to excellent results in terms of trajectory following and insensitivity to strong wind disturbances.

In fact, it has been demonstrated that this controller can withstand a variety of intense wind accelerations. It also ensures that static error performances are stable and precise. The PID-NN simulation results for the quad-rotor control strategy were provided to verify and ensure the controlled system's effectiveness in terms of quick and accurate responses.

Finally, it is to ensure that the work's goal has been met. In fact, when evolving in a windy environment, the controlled quad-rotor is able to protect itself from these problems without compromising its trajectory tracking, stability, or accuracy.

\section{REFERENCES}

[1] G. Singhal, B. Bansod, and L. Mathew, "Unmanned Aerial Vehicle Classification, Applications and Challenges: A Review," Preprints 2018110601 (doi: 10.20944/preprints201811.0601.v1) 2018.

[2] J. Ajmera and V. Sankaranarayanan, "Trajectory tracking control of a quad-rotor," 2015 International Conference on Control Communication \& Computing India (ICCC), Trivandrum, 2015, pp. 48-53, doi: 10.1109/ICCC.2015.7432868.

[3] C. Wang, W. Liu and M. Q. Meng, "Obstacle avoidance for quad-rotor using improved method based on optical flow," 2015 IEEE International Conference on Information and Automation, Lijiang, 2015, pp. 1674-1679, doi: 10.1109/ICInfA.2015.7279555.

[4] T. Üstünkök and M. Karakaya, "Effect of PSO Tuned P, PD, and PID Controllers on the Stability of a Quad-rotor," 2019 1st International Informatics and Software Engineering Conference (UBMYK), Ankara, Turkey, 2019, pp. 1-6, doi: 10.1109/UBMYK48245.2019.8965487. 
[5] J. J. Castillo-Zamora, K. A. Camarillo-GóMez, G. I. PéRez-Soto and J. RodríGuez-ReséNdiz, "Comparison of PD, PID and Sliding-Mode Position Controllers for V-Tail Quadcopter Stability,” in IEEE Access, vol. 6, pp. 38086-38096, 2018, doi: 10.1109/ACCESS.2018.2851223.

[6] H. S. Khan and M. B. Kadri, "Attitude and altitude control of quadrotor by discrete PID control and non-linear model predictive control," 2015 International Conference on Information and Communication Technologies (ICICT), Karachi, 2015, pp. 1-11, doi: 10.1109/ICICT.2015.7469486.

[7] H. Liu and G. Gao, "Dynamic modeling and analyzing for a novel Xquad-rotor," 2017 3rd International Conference on Computational Intelligence \& Communication Technology (CICT), Ghaziabad, 2017, pp. 1-4, doi: 10.1109/CIACT.2017.7977292.

[8] K. El Hamid, M. Mjahed, A. El Kari and H. Ayad, "Neural and Fuzzy Based Nonlinear Flight Control for an Unmanned Quad-rotor," International Review of Automatic Control (IREACO), vol. 11, no. 3, 2018.

[9] O. Doukhi, A. Fayjie and D.J. Lee, "Intelligent Controller Design for Quad-Rotor Stabilization in Presence of Parameter variations," Journal of Advanced Transportation, Vol. 2017, Article ID 4683912, 10 pages.

[10] M. Algabri, et al., "Wireless vision-based fuzzy controllers for moving object tracking using a quad-rotor," International Journal of Distributed Sensor Networks, vol. 13, no. 4, 2017.

[11] H.Housny, E.Chater and H. ElFadil, "Fuzzy PID Control Tuning Design Using Particle Swarm Optimization Algorithm for a Quadrotor," IEEE 5th International Conference on Optimization and Applications (ICOA), 2019.

[12] J. Xu, Y. Ma and J. Huang, "3D Path Tracking Controller Based on Fuzzy PID Optimized by PSO for Quad-rotor," 2019 Chinese Automation Congress (CAC), Hangzhou, China, 2019, pp. 3522-3527, doi: 10.1109/CAC48633.2019.8997208.

[13] T. Liu, Y. Chen, Z. Chen, H. Wu and L. Cheng, "Adaptive fuzzy fractional order PID control for 6-DOF quad-rotor," 2020 39th Chinese Control Conference (CCC), Shenyang, China, 2020, pp. 2158-2163, doi: 10.23919/CCC50068.2020.9188677.

[14] S. Bari, S. S. Zehra Hamdani, H. U. Khan, M. u. Rehman and H. Khan, "Artificial Neural Network Based Self-Tuned PID Controller for Flight Control of Quadcopter," 2019 International Conference on Engineering and Emerging Technologies (ICEET), Lahore, Pakistan, 2019, pp. 1-5, doi: 10.1109/CEET1.2019.8711864.

[15] M. Fatan, B. L. Sefidgari and A. V. Barenji, "An adaptive neuro PID for controlling the altitude of quadcopter robot," 2013 18th International Conference on Methods \& Models in Automation \& Robotics (MMAR), Miedzyzdroje, 2013, pp. 662-665, doi: 10.1109/MMAR.2013.6669989.

[16] S. Furukawa, S. Kondo, A. Takanishi and H. Lim, "Radial basis function neural network based PID control for quad-rotor flying robot," 2017 17th International Conference on Control, Automation and Systems (ICCAS), Jeju, 2017, pp. 580-584, doi: 10.23919/ICCAS.2017.8204300.

[17] A. Katiar, R. Rashdi, Z. Ali and U. Baig, "Control and stability analysis of quadcopter," 2018 International Conference on Computing, Mathematics and Engineering Technologies (iCoMET), Sukkur, 2018, pp. 1-6, doi: 10.1109/ICOMET.2018.8346419.

[18] R. Lopez, I. González-Hernández, S. Salazar, A. E. Rodriguez, J. J. Ordaz and A. Osorio, "Disturbance rejection for a Quadrotor aircraft through a robust control," 2015 International Conference on Unmanned Aircraft Systems (ICUAS), Denver, CO, 2015, pp. 409415, doi: 10.1109/ICUAS.2015.7152317.

[19] H. C. T. E. Fernando, A. T. A. De Silva, M. D. C. De Zoysa, K. A. D. C. Dilshan and S. R. Munasinghe, "Modelling, simulation and implementation of a quad-rotor UAV," 2013 IEEE 8th International Conference on Industrial and Information Systems, Peradeniya, 2013, pp. 207-212, doi: 10.1109/ICIInfS.2013.6731982.

[20] G. Jithu and P. R. Jayasree, "Quad-rotor modelling and control," 2016 International Conference on Electrical, Electronics, and Optimization Techniques (ICEEOT), Chennai, 2016, pp. 1167-1172, doi: 10.1109/ICEEOT.2016.7754868.

[21] M. Walid, N. Slaheddine, A. Mohamed and B. Lamjed, "Modelling, Identification and Control of a Quad-rotor UAV," 2018 15th International Multi-Conference on Systems, Signals \& Devices (SSD), Hammamet, 2018, pp. 1017-1022, doi: 10.1109/SSD.2018.8570512.

[22] M. Ardema, "Newton-Euler Dynamics," Springer Science and Business Media, Inc, 2005.

[23] Y. Chen, Y. He and M. Zhou, "Decentralized PID neural network control for a quad-rotor helicopter subjected to wind disturbance," Journal of Central South University, Vol. 22, No. 1, pp. 168-179, 2015.
[24] L. Ding and Z. Wang, "A Robust Control for an Aerial Robot Quadrotor under Wind Gusts," Journal of Robotics, Vol. 2018, pp. 1-8, 2018.

[25] G. Perozzi, D. Efimov, J. Biannic, L. Planckaert and P. Coton, "Wind estimation algorithm for quad-rotors using detailed aerodynamic coefficients," 2018 Annual American Control Conference (ACC), Milwaukee, WI, 2018, pp. 1921-1926, doi: 10.23919/ACC.2018.8431879.

[26] Y. Guo, B. Jiang and Y. Zhang, "A novel robust attitude control for quad-rotor aircraft subject to actuator faults and wind gusts," IEEE/CAA Journal of Automatica Sinica, Vol. 5, No. 1, pp. 292-300, 2018.

[27] R. T. Palomaki, N. T. Rose, M. van den Bossche, T. J. Sherman, and S. F. J. De Wekker, "Wind estimation in the lower atmosphere using multirotor aircraft," J. Atmos. Ocean. Technol., vol. 34, no. 5, pp. 11831191, May 2017.

[28] Z. He and L. Zhao, "A Simple Attitude Control of Quad-rotor Helicopter Based on Ziegler-Nichols Rules for Tuning PD Parameters," The Scientific World Journal, Vol. 2014, Article ID 280180, 13 pages, 2014.

[29] K. J. Astrom and T. Hagglund, "Revisiting the Ziegler-Nichols step response method for PID control," Elsevier, Journal of Process Control, Vol. 14, pp. $635-650,2004$. 